\title{
Changes of Osteopontin Distribution and Matrix Mineralization during Remodeling in Experimental Bone Formation
}

\author{
Kazuharu Irie $^{1}$, Hideyuki Takeishi², Eichi Tsuruga' ${ }^{1}$, Yasunori Sakakura1, \\ Shuichi Nomura ${ }^{3}$, Hidehiro Ozawa ${ }^{4}$, Sadakazu Ejiri ${ }^{5}$ and Toshihiko Yajima ${ }^{1}$ \\ ${ }^{1}$ Department of Oral Anatomy, Health Sciences University of Hokkaido School of Dentistry, Hokkaido, ${ }^{2}$ Department of \\ Oral Biological Science, Division of Dysphagia Rehabilitation, Niigata University Graduate School of Medical and Dental \\ Sciences, Niigata, ${ }^{3}$ Department of Oral Health Science, Division of Oral Health in Aging and Fixed Prosthodontics, Niigata \\ University Graduate School of Medical and Dental Sciences, Niigata, ${ }^{4}$ Institute for Dental Science and Department of Oral \\ Anatomy, Matsumoto Dental University, Shiojiri and ${ }^{5}$ Department of Tissue Regeneration and Reconstruction, Division of \\ Anatomy and Cell Biology of the Hard tissue,Niigata University Graduate School of Medical and Dental Sciences, Niigata
}

Received February 20, 2002; accepted March 25, 2002

\begin{abstract}
When bone is rapidly induced by recombinant human bone morphogenetic protein-2 (rhBMP-2), more noncollagenous proteins (NCPs) are accommodated among the collagen meshwork. These NCPs are then removed, resulting in highly mineralized mature bone. However, few reports have focused on changes in the bone matrix of rhBMP-2-induced bone. In the present study, rhBMP-2 with an artificial carrier was implanted over bone defects in rat calvariae, and changes in the distribution of osteopontin (OPN), the degree of mineralization and speed of bone formation were investigated histochemically and radiographically. Domeshaped areas of newly formed bone observed at postoperative week 2 were

intensely immunoreactive for OPN and intensely labeled with calcein, but were not as radiopaque as the preexisting bone. At postoperative week 8 , intense immunoreactivity was detected only on cement lines and in small discrete areas on the flattened domes. The matrix was as radiopaque as, and indistinguishable from, the preexisting bone. Only thin linear labeling of calcein was found on the bone surface. These findings suggest that, in rhBMP-2 induced bone, production of OPN is increased when the rate of bone formation is high, and that OPN produced in the early stage of bone formation is removed during bone remodeling to create a highly mineralized mature bone matrix.
\end{abstract}

Key words: Bone remodeling, Mineralization, Noncollagenous proteins, Osteopontin, rhBMP-2

\section{Introduction}

Bone morphogenetic proteins (BMPs), members of the transforming growth factor (TGF)-beta superfamily, are known to be included among a number of factors that induce rapid bone formation. Recombinant techniques have made BMPs accessible for biological and preclinical evaluation (reviewed in [27]). Local administration of recombinant human BMP-2 (rhBMP-2) induces ectopic bone formation and improves the healing of fractures and various bone defects in animals $[1,15,16,28]$. Such studies of rhBMP-2 have

Correspondence to: Kazuharu Irie, D.D.S., Ph.D., Department of Oral Anatomy, Health Sciences University of Hokkaido School of Dentistry, Ishikari-Tobetsu, Hokkaido 061-0293, Japan. demonstrated an increased rate of bone formation at an early stage of osteogenesis and regeneration. BMP expression has also been demonstrated during fracture healing [8]. BMPs accelerate migration, proliferation and differentiation of bone-forming cells, and stimulate matrix secretion; up-regulation of gene expression and secretion of matrix proteins such as type I collagen and noncollagenous proteins (NCPs) have been reported $[4,10,14]$. The collagen fibers in lamellar bone are usually oriented in a preferential direction and closely packed, whereas in woven bone they appear as irregular bundles and have comparatively wide interfiber spaces. NCPs have been demonstrated in the spaces between collagen fibers $[5,13,17,18]$. Biologically, these matrix proteins mediate various events involving cells and the bone matrix, including mineralization during bone formation, and 
subsequent bone remodeling. In this context, the distribution of matrix proteins implies that biological signalling occurs in bone. However, few reports have focused on the changes occurring in induced bone matrix, especially changes in the distribution of NCPs, the degree of bone mineralization, and the speed of bone formation.

Therefore, the present study was designed to clarify the relationship between the distribution of osteopontin (OPN), one of the major NCPs in bone, and bone mineralization with reference to the speed of bone formation and remodeling in rhBMP-2-induced bone.

\section{Materials and Methods}

Twelve 6-week-old male Wistar rats were anesthetized with diethyl ether and an intraperitoneal injection of $8 \%$ chloral hydrate $(0.5 \mathrm{ml} / 100 \mathrm{~g}$ body weight $)$. After shaving the head, a $3-\mathrm{cm}$ midsagittal incision was made, and the periosteum and subcutaneous tissue were reflected to expose the skull. Bone defects $1.0 \mathrm{~mm}$ in diameter and $0.5 \mathrm{~mm}$ deep were made on both parietal bones using a round dental burr (\#3, $1.0 \mathrm{~mm}$ diameter) operated at low speed while irrigating the site with sterilized physiological saline. Five micrograms of rhBMP-2 in a carrier made of polylactic acid-polyglycolic acid copolymer gelatin sponge complex (PGS) was placed over each of the two bone defects in the skull. These defects were to communicate with bone marrow and to provide the cellular components that participate in bone formation and remodeling. Then the periosteum and subcutaneous tissues were replaced, and the surgical wounds were closed with sutures. The animals were killed 2 and 8 weeks after surgery. Principles of laboratory animal care were followed and animal experimentation was in compliance with the "Guidelines for the Care and Use of Laboratory Animals in Health Sciences University of Hokkaido".

\section{Histological and histochemical studies}

After anesthesia with an injection of pentobarbital, the rats were fixed by perfusion with $4 \%$ paraformaldehyde in $0.1 \mathrm{M}$ cacodylate buffer ( $\mathrm{pH}$ 7.4). The skulls were quickly dissected and immersed in the same fixative overnight. The specimens were rinsed in $0.1 \mathrm{M}$ cacodylate buffer and decalcified with $4.13 \%$ EDTA for 3 weeks at $4^{\circ} \mathrm{C}$. The skulls were then divided along the sagittal suture, dehydrated in a series of increasing concentrations of ethanol, and embedded in paraffin. Sagittal sections, $5.0 \mu \mathrm{m}$ thick through the middle part of the defect, were selected from serial sections and stained with hematoxylin and eosin. Certain sections for immunohistochemical examination were treated with $0.3 \%$ hydrogen peroxide in methanol for $30 \mathrm{~min}$, followed by incubation for $1 \mathrm{hr}$ with PBS containing 5\% skim milk. Then the specimens were incubated with goat anti-rat OPN (Dr. M. C. Farach-Carson, University of Texas at Houston) at a dilution of 1:200 in PBS for $2 \mathrm{hr}$, followed by incubation with anti-goat Igs (Kirkegaard \& Perry laboratories Inc., Gaithersburg, MD) at a dilution of 1:100 in PBS for $1 \mathrm{hr}$. Immunoreactivity was visualized by incubation in $0.05 \mathrm{M}$
Tris-HCl buffer ( $\mathrm{pH} 7.6$ ) containing $0.01 \%$ 3,3'-diaminobenzidine and $0.002 \%$ hydrogen peroxide. All incubations were done at room temperature. The sections were counterstained with hematoxylin. With a control solution of PBS without anti-rat OPN, no significant reaction on the sections was found.

\section{Calcein labeling and contact microradiography}

Two of the experimental animals at each time point were subcutaneously injected with calcein $(10 \mathrm{mg} / \mathrm{kg}$; Wako, Osaka, Japan) [12] $24 \mathrm{hr}$ prior to sacrifice. Following decapitation, the skulls were fixed in $70 \%$ ethanol for 7 days. The specimens were then divided along the sagittal suture, dehydrated with ethanol and embedded in methylmethacrylate (Wako, Tokyo, Japan) without decalcification. The blocks were unilaterally ground up to the middle of the bone defect. The calcein labeling was observed by mounting the midsagittal surface of the ground blocks on the stage of a confocal laser scanning microscope (CLSM; LSM-GB200; Olympus, Tokyo, Japan). The blocks were then ground to a thickness of $90 \mu \mathrm{m}$. Contact microradiographs were taken with a SOFRON SRO-405 instrument (Sofron, Tokyo, Japan), employing Kodak SO-343 high-resolution film (Eastman Kodak, Rochester, NY). Irradiation was done at $14.5 \mathrm{kV}$ and $5.0 \mathrm{~mA}$ for $25 \mathrm{~min}$.

\section{Results}

Along the outline of the rhBMP-2 implant, an obvious dome-shaped area of bone was observed over the defect at postoperative week 2 . Inside the dome, the residues of the PGS carrier and islands of newly formed bone were distributed among the cellular components. Trabecular bone extended both from the dome wall and preexisting bone towards the inside to connect the bone islands. These newly formed bones were woven bones with eosinophilic characteristic (Fig. 1A). At postoperative week 8, the dome had decreased in height, sloping gently down to merge with the skull. Bone matrix of the dome was as compact as preexisting calvaria. The bone marrow extended under the flattened dome, and the PGS carrier was no longer obvious (Fig. 1B). At week 2, microradiography showed radiolucent punchedout areas in the dome and bone islands, giving the appearance of a fine meshwork of mineralized matrix, which was more radiolucent than the preexisting calvaria (Fig. 2A). At week 8 , the flattened dome was as radiopaque as the preexisting bone and indistinguishable from it (Fig. 2B).

Immunoreactivity for OPN was intense on the matrix of the dome-shaped bone and bone islands inside it at postoperative week 2 (Fig. 3A, B). However, this immunoreactivity seemed to consist of accumulations of immunoreactive patches. From the size and the distribution of the patches, these seemed to correspond with radiolucent punched-out areas in microradiogram. At week 8 , small areas and discrete lines within the flattened dome were immunolabeled for OPN (Fig. 3C). The distribution and intensity of the immunoreactivity in the flattened dome at postoperative week 8 


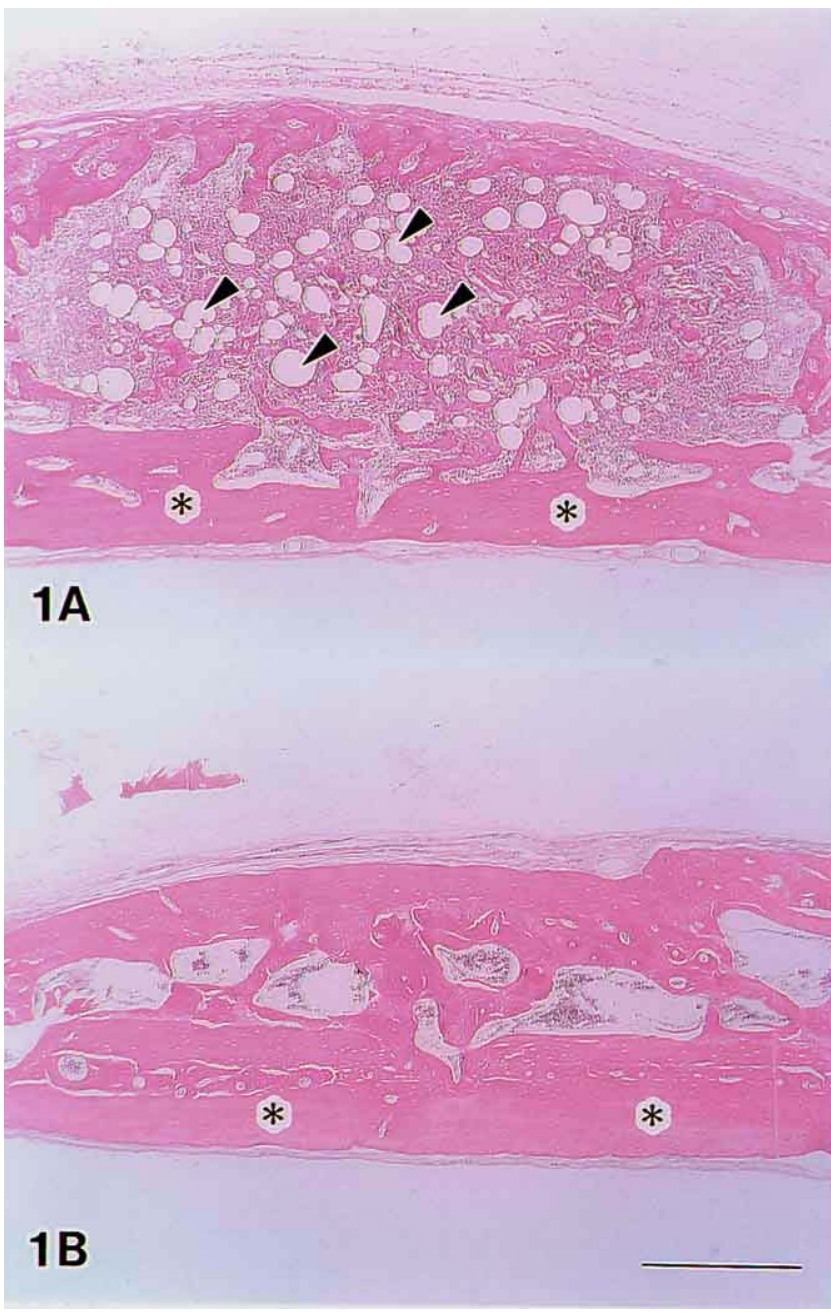

Fig. 1. Histological appearance of calvaria after implantation of rhBMP-2 with a PGS carrier. (A) Along the outline of the implant, a dome-shaped area of bone is obvious at postoperative week 2 . Among the cellular components, newly formed bone islands and residues of the PGS carrier (arrowheads) can be seen inside the dome. These newly formed bones are eosinophilic. (B) At week 8, the dome appears flat and slopes gently down to the calvaria, giving an image of calvarial thickening. Bone matrix of the dome is as compact as preexisting calvaria (asterisks). Bar $=500 \mu \mathrm{m}$.

were similar to those of the preexisting bone (Fig. 3D).

Calcein labeling produced broad staining of the newly formed dome and bone islands at week 2, while in the preexisting bone the labeling was detected as thin lines at the surface of the calvaria and the surface facing the bone marrow (Fig. 4A). At week 8, linear calcein labeling was observed over the surface of both the flattened dome and preexisting bone (Fig. 4B).

\section{Discussion}

Previously, we showed that rhBMP-2-induced domeshaped areas of bone underwent remodeling that resulted in a decrease of height [24]. The present study demonstrated
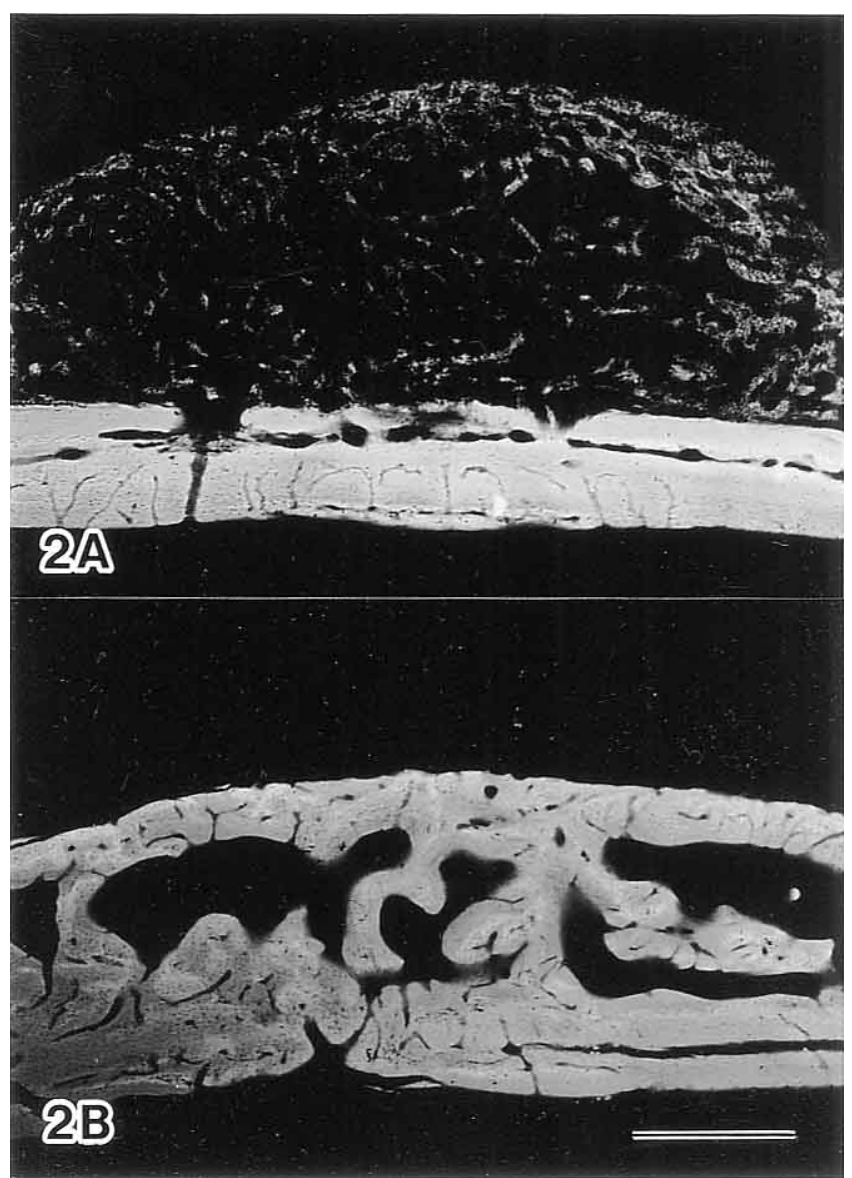

Fig. 2. Contact radiomicrographs of calvaria after implantation of rhBMP-2 with the PGS carrier. (A) At postoperative week 2, a newly formed area of dome-shaped bone and bone islands within it show radiolucent patches in a radiopaque matrix, suggesting that the newly formed bone is not as radiopaque as the preexisting calvaria. (B) At postoperative week 8, the flat dome is as radiopaque as the preexisting calvaria, and indistinguishable from it. Bar $=500 \mu \mathrm{m}$.

that the dome-shaped bone contained a large amount of OPN, and had been slowly replaced by highly mineralized mature bone during remodeling. The cement lines associated with OPN at week 8 in this study were also evidence of bone remodeling. The discrete areas showing intense immunoreactivity appeared to correspond to the areas where initially formed bone matrix remained during remodeling.

OPN was demonstrated in the spaces between collagen fibers, suggesting that bone containing tightly packed collagen has less OPN, whereas looser matrices such as woven bone have substantially more OPN (reviewed in [20]). Indeed, the weakest immunoreactions for OPN demonstrated by immunoelectron microscopy were over regions rich in mineralized collagen. Since looser packing of collagen, a characteristic feature of woven bone, appears when bone is formed very rapidly - for example in the early stage in development and in fracture healing - the packing density of collagen and the distribution of OPN may reflect the speed of bone formation. In fact, high expression of OPN in devel- 


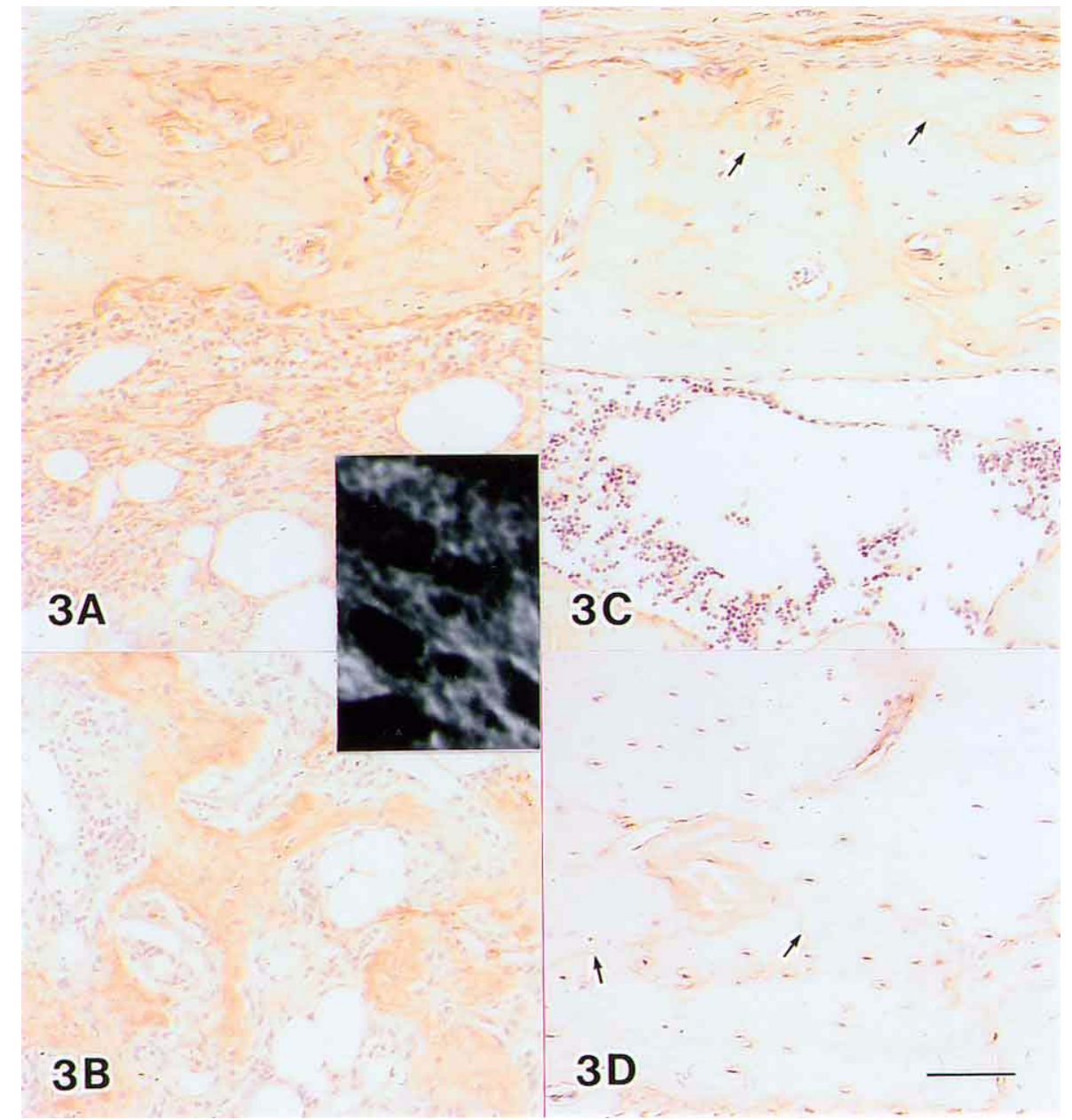

Fig. 3. Immunohistochemical localization of osteopontin $(\mathrm{OPN})$ in calvaria after implantation of rhBMP- 2 with the PGS carrier. Bone matrix of dome-shaped bone (A) and bone islands inside it (B) at postoperative week 2 are intensely immunoreactive for OPN. Note matrix consists of immunoreactive patches, especially in bone islands. These patches correspond to radiolucent punched-out areas in microradiogram shown in insert. Bone matrix of the flat dome at postoperative week 8 shows generally weak immunoreactivity $(\mathbf{C})$ and is similar to the immunoreactivity in the preexisting bone (D). Discrete lines (arrows) in the bone matrix, corresponding to cement lines, are intensely immunoreactive in both the flat dome and the preexisting bone. Insert, high magnification of radiogram shown as $2 \mathrm{~A}$. Bar $=100 \mu \mathrm{m}$.

oping limbs and mandible has been demonstrated [9, 21, 23, 26, 29]. Moreover, the spatial organization and relatively wide spaces in the collagen meshwork of woven bone could accommodate more NCPs. As well as in developing bones, intense immunoreactivity for OPN has also been demonstrated in experimentally induced woven bone after direct administration of basic fibroblast growth factor into rat bone marrow [2]. Such woven bone is usually not as mineralized as lamellar bone. During development or fracture healing, woven bone is replaced by mature lamellar bone in the process of bone remodeling. In this study, intense immunoreaction for OPN was found on the newly formed bone matrix at week 2, suggesting rapid bone formation with looser packing of collagen induced by rhBMP-2. Indeed, broad incorporation of calcein labeling in newly formed bone during this period indicated a high rate of mineralization, and thus bone formation. On the other hand, at week 8 , intense immunoreactivity for OPN was restricted to cement lines and small discrete areas, and the overall pattern of immunoreactivity resembled that of preexisting bone. This variability of OPN immunolabeling is comparable to that observed in normal bone. Calcein incorporation was very limited at this stage, and only linear labeling was evident on the external and internal surfaces of bone, suggesting that bone formation was much slower than that at week 2 . These data, when consider the discussion above, suggest that the OPN content in bone matrix is influenced by the speed of bone formation and the packing density of collagen. A relationship between collagen packing density and the amount of NCPs has also been documented in tooth cementum, a tissue with general similarities to bone. In cementum, as in this study, the rate of formation is an important determinant of the relative proportions of NCPs and collagen [3]. A large quantity of OPN, together with all other NCPs, in initially formed bone may rapidly provide a space, with some rigidity, for replacing mature lamellar bone. Moreover, most of the bone matrix 


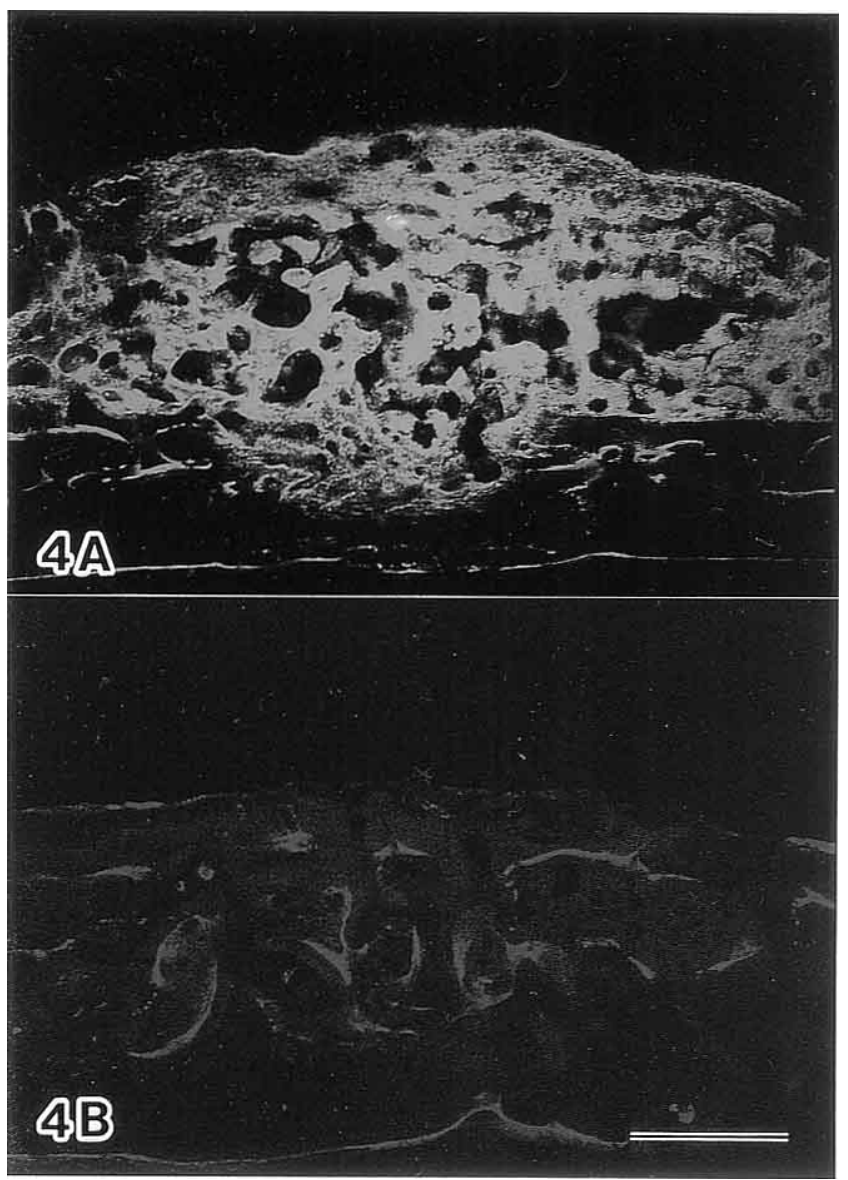

Fig. 4. Confocal laser scanning micrographs of calvaria with calcein labeling after implantation of rhBMP-2 with the PGS carrier. (A) At postoperative week 2 , calcein is broadly integrated into the newly formed bone, labeling the whole matrix of the dome-shaped bone and bone islands. In the preexisting bone, labeling is detected as thin lines at the surface of the calvaria and the surface facing the bone marrow. (B) At postoperative week 8, linear calcein labeling is evident over the surface of both the flattened dome and preexisting bone, as well as the surface facing the bone marrow. Bar=500 $\mu \mathrm{m}$.

with a high content of OPN will eventually undergo remodeling. Together with reports suggesting a possible role of OPN in the migration and cell-matrix adhesion of osteoclasts $[7,11,22,25]$, OPN may facilitate osteoclastic bone resorption and subsequent bone remodeling.

Interestingly, the patch-like pattern of intense immunolabeling for OPN at week 2 appeared consistent with a punched-out pattern of radiolucency, suggesting an inhibitory function of OPN in matrix mineralization. Immunoelectron microscopy during the early mineralization process demonstrated an association of OPN with mineralizing foci and small globular masses among the collagen fibrils. Moreover, most of the intense immunolabeling for OPN disappeared when newly formed bone underwent remodeling to become highly mineralized mature bone, consistent with the idea that OPN helps regulate the mineralization of bone matrix $[6,19]$.
It is noteworthy that the outline of the dome-shaped bone area seen at week 2 corresponded to the outline of the BMP implant. Since bone matrix inside the implant was more radiolucent than that at the periphery of the implant at this point, it was considered that bone formation occurred at the periphery of the BMP implant where migrated mesenchymal cells would have encountered a specific concentration of diffused rhBMP-2.

We conclude that in rhBMP-2-induced bone, production of OPN is increased when the rate of bone formation is high, and that OPN produced at an early stage of bone formation is removed during bone remodeling to achieve highly mineralized mature bone: a situation resembling that in normal bone.

\section{Acknowledgments}

The authors wish to thank Dr. M. C. Farach-Carson (University of Texas at Houston) for donation of antibodies, and Yamanouchi Pharmaceutical Co. Ltd. for kindly providing rhBMP-2. This work was supported by The Akiyama Foundation and a Grant-in-Aid for Scientific Research (13307059).

\section{References}

1. Alpaslan, C., Irie, K., Takahashi, K., Ohashi, N., Sakai, H., Nakajima, T. and Ozawa, H.: Long-term evaluation of recombinant human bone morphogenetic protein-2 induced bone formation with a biologic and synthetic delivery system. Br. J. Oral Maxillofac. Surg. 34; 414-418, 1996.

2. Amizuka, N., Yamada, M., Watanabe, J. I., Hoshi, K., Fukushi, M., Oda, K., Ikehara, Y. and Ozawa, H.: Morphological examination of bone synthesis via direct administration of basic fibroblast growth factor into rat bone marrow. Microsc. Res. Tech. 41; 313322, 1998.

3. Bosshardt, D. D., Zalzal, S., McKee, M. D. and Nanci, A.: Developmental appearance and distribution of bone sialoprotein and osteopontin in human and rat cementum. Anat. Rec. 250; 13-33, 1998.

4. Chen, D., Harris, M. A., Rossini, G., Dunstan, C. R., Dallas, S. L., Feng, J. Q., Mundy, G. R. and Harris, S. E.: Bone morphogenetic protein 2 (BMP-2) enhances BMP-3, BMP-4, and bone cell differentiation marker gene expression during the induction of mineralized bone matrix formation in cultures of fetal rat calvarial osteoblasts. Calcif. Tissue Int. 60; 283-290, 1997.

5. Chen, J., McKee, M. D., Nanci, A. and Sodek, J.: Bone sialoprotein mRNA expression and ultrastructural localization in fetal porcine calvarial bone: comparisons with osteopontin. Histochem. J. 26; 67-78, 1994.

6. Denhardt, D. T. and Guo, X.: Osteopontin: a protein with diverse functions. Faseb J. 7; 1475-1482, 1993.

7. Dodds, R. A., Connor, J. R., James, I. E., Rykaczewski, E. L., Appelbaum, E., Dul, E. and Gowen, M.: Human osteoclasts, not osteoblasts, deposit osteopontin onto resorption surfaces: an in vitro and ex vivo study of remodeling bone. J. Bone Miner. Res. $10 ; 1666-1680,1995$.

8. Fujimoto, T., Kitazawa, R., Maeda, S., Mizuno, K. and Kitazawa, S.: BMP-3 mRNA expression during endochondral ossification of mouse bone tissue. Acta Histochem. Cytochem. 34; 1-7, 2001.

9. Hirakawa, K., Hirota, S., Ikeda, T., Yamaguchi, A., Takemura, T., Nagoshi, J., Yoshiki, S., Suda, T., Kitamura, Y. and Nomura, 
S.: Localization of the mRNA for bone matrix proteins during fracture healing as determined by in situ hybridization. J. Bone Miner. Res. 9; 1551-1557, 1994.

10. Imamura, T., Nagatsuka, H., Inoue, M., Murata, M. and Nagai, N.: Developmental expression of osteopontin and osteocalcin mRNA during ectopic bone formation by recombinant human BMP-2. J. Okayama Dent. Society 14; 1-13, 1995. (in Japanese)

11. Ingram, R. T., Clarke, B. L., Fisher, L. W. and Fitzpatrick, L. A. Distribution of noncollagenous proteins in the matrix of adult human bone: evidence of anatomic and functional heterogeneity. J. Bone Miner. Res. 8; 1019-1029, 1993.

12. Irie, K., Ozawa, H. and Yajima, T.: The histochemical and cytochemical changes from formative to resorptive osteocytes. Acta Histochem. Cytochem. 33; 385-391, 2000.

13. Irie, K., Zalzal, S., Ozawa, H., McKee, M. D. and Nanci, A.: Morphological and immunocytochemical characterization of primary osteogenic cell cultures derived from fetal rat cranial tissue. Anat. Rec. 252; 554-567, 1998.

14. Lecanda, F., Avioli, L. V. and Cheng, S. L.: Regulation of bone matrix protein expression and induction of differentiation of human osteoblasts and human bone marrow stromal cells by bone morphogenetic protein-2. J. Cell Biochem. 67; 386-396, 1997.

15. Marukawa, E., Asahina, I., Oda, M., Alam, M. I. and Enomoto, S.: Bone regeneration using recombinant human bone morphogenetic protein-2 (rhBMP-2) in alveolar defects of primate mandibles. Br. J. Oral Maxillofac. Surg. 39; 452-459, 2001.

16. Matin, K., Nakamura, H., Irie, K., Ozawa, H. and Ejiri, S.: Impact of recombinant human bone morphogenetic protein- 2 on residual ridge resorption after tooth extraction: an experimental study in the rat. Int. J. Oral Maxillofac. Implants 16; 400-411, 2001.

17. McKee, M. D. and Nanci, A.: Postembedding colloidal-gold immunocytochemistry of noncollagenous extracellular matrix proteins in mineralized tissues. Microsc. Res. Tech. 31; 44-62, 1995.

18. McKee, M. D., Glimcher, M. J. and Nanci, A.: High-resolution immunolocalization of osteopontin and osteocalcin in bone and cartilage during endochondral ossification in the chicken tibia. Anat. Rec. 234; 479-492, 1992.

19. McKee, M. D., Nanci, A., Landis, W. J., Gotoh, Y., Gerstenfeld, L. C. and Glimcher, M. J.: Developmental appearance and ultrastructural immunolocalization of a major $66 \mathrm{kDa}$ phosphoprotein in embryonic and post-natal chicken bone. Anat. Rec. 228; 77-92,
1990.

20. Nanci, A.: Content and distribution of noncollagenous matrix proteins in bone and cementum: relationship to speed of formation and collagen packing density. J. Struct. Biol. 126; 256-269, 1999.

21. Nomura, S., Wills, A. J., Edwards, D. R., Heath, J. K. and Hogan, B. L.: Developmental expression of 2 ar (osteopontin) and SPARC (osteonectin) RNA as revealed by in situ hybridization. J. Cell Biol. 106; 441-450, 1988.

22. Reinholt, F. P., Hultenby, K., Oldberg, A. and Heinegard, D.: Osteopontin - a possible anchor of osteoclasts to bone. Proc. Nat. Acad. Sci. US A 87; 4473-4475, 1990.

23. Sasano, Y., Zhu, J. X., Kamakura, S., Kusunoki, S., Mizoguchi, I. and Kagayama, M.: Expression of major bone extracellular matrix proteins during embryonic osteogenesis in rat mandibles. Anat. Embryol. (Berl) 202; 31-37, 2000.

24. Takeishi, H., Irie, K., Okuda, K., Ozawa, H., Yajima, T. and Ejiri, S.: Molded bone augmentation by a combination of barrier membrane and recombinant bone morphogenetic protein-2. Oral Dis. 7; 281-286, 2001.

25. Terai, K., Takano-Yamamoto, T., Ohba, Y., Hiura, K., Sugimoto, M., Sato, M., Kawahata, H., Inaguma, N., Kitamura, Y. and Nomura, S.: Role of osteopontin in bone remodeling caused by mechanical stress. J. Bone Miner. Res. 14; 839-849, 1999.

26. Weinreb, M., Shinar, D. and Rodan, G. A.: Different pattern of alkaline phosphatase, osteopontin, and osteocalcin expression in developing rat bone visualized by in situ hybridization. J. Bone Miner. Res. 5; 831-842, 1990.

27. Wozney, J. M., Rosen, V., Celeste, A. J., Mitsock, L. M., Whitters, M. J., Kriz, R. W., Hewick, R. M. and Wang, E. A.: Novel regulators of bone formation: molecular clones and activities. Science 242; 1528-1534, 1988

28. Yasko, A. W., Lane, J. M., Fellinger, E. J., Rosen, V., Wozney, J. M. and Wang, E. A.: The healing of segmental bone defects, induced by recombinant human bone morphogenetic protein (rhBMP-2). A radiographic, histological, and biomechanical study in rats. J. Bone Joint Surg. Am. 74; 659-670, 1992.

29. Zhu, J. X., Sasano, Y., Takahashi, I., Mizoguchi, I. and Kagayama, M.: Temporal and spatial gene expression of major bone extracellular matrix molecules during embryonic mandibular osteogenesis in rats. Histochem. J. 33; 25-35, 2001. 